\title{
Oral Session 1: Neural Adaptation and Sensory Transduction
}

\section{1-1 [\#3019]}

\section{Experimental measurement of utricle dynamic response}

J.W. Grant ${ }^{\mathrm{a}}$, M.D. Dunlap ${ }^{\mathrm{b}}$ and C.E. Spoon ${ }^{\mathrm{c}}$

${ }^{a}$ Departments of Biomedical Engineering and Engineering Science and Mechanics, VA Tech, Blacksburg, VA, USA.E-mail: jgrant@vt.edu

${ }^{\mathrm{b}}$ Department of Biomedical Engineering, VA Tech, Blacksburg, VA, USA

${ }^{\mathrm{c}}$ Departments of Biomedical Engineering and Engineering Science and Mechanics, VA Tech, Blacksburg, VA, USA

Introduction: The utricle of the red-eared turtle was subjected to forced sinusoidal oscillations across various frequencies (10-125 Hz) and amplitudes (5$15 \mu \mathrm{m}$ ) to determine utricle dynamic characteristics.

Experiment: After removing the utricle, the overlying utricle membrane was trimmed away to expose the otoconial crystals layer (CL) without disturbing this layer or the underlying neural epithelial layer (EL). The utricle was maintained under compatible solution, placed on a glass slide, and made parallel to the slide using glass chips. Three single strands of dental floss were use to rigidly attached the EL to the glass slide, while not impairing the natural CL displacement. A piezoelectric-actuated platform was fitted into the stage of the microscope and created controlled vibrations along the medial-lateral direction of the utricle. The CL surface crystal's displacement was measured through the microscope with high-speed video (1500 fps, resolution $=144 \mathrm{~nm} /$ pixel). A reference point outside macula was filmed under identical amplitude and frequency inputs to evaluate any small displacement relative to the glass slide motion. Cross correlation in a Matlab program determined CL and reference point displacements. Maximum displacement amplitudes of the CL and reference point relative to the piezoelectric actuator were used to determine the Amplitude ratio of the CL to reference point.

Results: Fitting a single degree of freedom model to measured values $(n=9)$ of amplitude ratios determined the utricle's natural frequency of $349 \mathrm{~Hz}(\sigma=$ $95)$ with a damping ratio of $1.44(\sigma=0.22)$. No amplitude or frequency dependence of damping or natural frequency was seen.

\section{1-2 [\#3039]}

The effect of microgravity on mRNA expression in the vestibular endorgans: Comparison of the 90-day and 15-day space flight

Y. Takumi ${ }^{\mathrm{a}}$, T. Oguchi ${ }^{\mathrm{a}}$, N. Suzuki ${ }^{\mathrm{a}}$, S. Nishio ${ }^{\mathrm{a}}$, R. Boyle $^{\mathrm{b}}$ and S. Usami ${ }^{\mathrm{a}}$

${ }^{a}$ Department of Otorhinolaryngology, Shinshu University School of Medicine, 3-1-1, Asahi, Matsumoto, 3908621, Japan.E-mail: takumi@shinshu-u.ac.jp

${ }^{\mathrm{b}}$ BioVIS Technology Center, Mail Stop 239-11, Ames Research Center, NASA, Moffett Field, CA 94035, USA

Significant decrease of vestibular inputs during the space flight is one of the major reasons for space motion sickness. In space, astronauts adapt normally to the microgravity environment within a few days. Indeed the central nervous system has a central role of adaptation, but the vestibular endorgans are believed to also show adaptation. Previous space flight study revealed the obvious change of synaptic arrangement in the rat vestibular endorgans [1]. Therefore, it is interesting and important to investigate the gene expression patterns in the vestibular endorgans during space flight. In this study, mRNAs from the mouse vestibular endorgans in two different space flight groups were analyzed. 
90-day flight group: The samples were provided by Biospecimen Sharing Program (TSP) of Mice

Drawer System (STS-128/129). Only three mice survived for 90 days in the mission to the International Space Station. The vestibular endorgans were dissected out from the temporal bones under RNA later solution and mRNAs were extracted.

15-day flight group: The samples were provided by BSP of STS-131. Tissues were extracted same as above.

Each extraction was quality checked and applied for DNA microarray. In each sample genes were selected from the gene profile that exhibited either an up $(>$ 2 -fold) or down $(<0.5)$ regulation. In 90-day flight group, 71 up- regulated and 43 down-regulated genes were detected. In contrast, the gene expression patterns were quite different in the 15-day flight group. These genes might be key molecules altered by microgravity and these analyses might shed light on the time course and underlying mechanisms driving vestibular adaptation during the space flight.

\section{Reference}

[1] M.D. Ross, Acta Otolaryngol 120 (2000), 490-499.

\section{1-3 [\#3004]}

\section{Neurovestibular adaptation in the utricular otolith following extended periods of hypergravity exposure and re-adaptation to $1 \mathrm{G}$}

\author{
R. Boyle ${ }^{\mathrm{a}}$, Y. Popova ${ }^{\mathrm{a}}$, J. Varelas ${ }^{\mathrm{a}}$ and A. Kondrachuk ${ }^{\mathrm{b}}$ \\ ${ }^{a}$ BioVIS Center, NASA Ames Research Center, Moffett \\ Field, CA, USA \\ ${ }^{\mathrm{b}}$ Inst. Physics, Kiev, Ukraine
}

Space flight introduces a novel sensory environment and initiates adaptive mechanisms within the nervous system. Humans typically adapt to $\mu \mathrm{G}$ within $2-4$ days and subsequently must re-adapt upon return to $1 \mathrm{G}$. Here we ask: does the transfer from $1 \mathrm{G}$ to $3 \mathrm{G}$ impart the opposite effects on changes of gravitational sensitivity seen following $\mu \mathrm{G}$ exposure? Do the effects accompanying transfer from the $3 \mathrm{G}$ to the $1 \mathrm{G}$ conditions resemble in part (as an analog) the transfer from $1 \mathrm{G}$ to the $\mu \mathrm{G}$ ? Adult toadfish were exposed to $3 \mathrm{G}$ for $1-32$ days. Re-adaptation to $1 \mathrm{G}$ was studied after $3 \mathrm{G}$ exposure of 4- and 16-days followed by 1-8 days of recovery.
Directional selectivity to translational and tilt stimuli were well characterized in typically $>60$ afferents in each subject. Results show a biphasic response to $3 \mathrm{G}$ : an initial sensitivity increase (3- and 4-day), similar in magnitude to that observed in utricular afferents upon return to Earth, followed by a transition through normal sensitivity to a significant decrease at 16-32 day exposure. Return to control values following $3 \mathrm{G}$ exposure is $\sim 4-8$ days. As an internal control horizontal canal afferents respond normally to yaw rotation. On-Center Controls ( $228 \%$ s rotation about Earth vertical) at 4 - and 16-days respond normally for both utricular and canal afferents. Utricular sensitivity is strongly regulated by altered gravity signal, beginning within hours to days, and transition from hyper-G to normal gravity resembles the transfer from $1 \mathrm{G}$ to $\mu \mathrm{G}$, and might be used as an analog ground-based model.

Acknowledgement: Support contributed by: NASA 03-OBPR-04.

\section{1-4 [\#3045]}

\section{Spaceflight-induced plasticity of utricular hair cell synapses}

L.F. Hoffman ${ }^{\mathrm{a}}$, D.R. Sultemeier ${ }^{\mathrm{a}}$, P.M. Quinones ${ }^{\mathrm{b}}$, G.A. Zampighi ${ }^{\mathrm{b}}$ and F.E. Schweizer ${ }^{\mathrm{b}}$

${ }^{a}$ Department of Surgery/Head and Neck, David Geffen School of Medicine at UCLA, Los Angeles, CA 90095, USA

${ }^{\mathrm{b}}$ Department of Neurobiology, David Geffen School of Medicine at UCLA, Los Angeles, CA 90095, USA

Previous ultrastructural studies indicated that spaceflight resulted in upregulation of synapse density in utricular hair cells; however, it was not tested whether this could be generalized to hair cells distributed across the utricular topography. In the present study we addressed this issue using double-label immunohistochemistry and volumetric image analysis that facilitated broad topologic sampling, and report our findings from the medial extrastriola region. Specimens were obtained from C57BL/6J mice flown aboard Discovery during STS-131 (flight group), and from age-matched ground controls (control group). Temporal bones from flight specimens were harvested within $\mathrm{R}+5 \mathrm{hrs}$ and immersion-fixed (4\% paraformaldehyde); control specimens were similarly obtained. Immunohistochemistry was performed on microdissected epithelia to label synaptic ribbons (anti-CtBP2) within hair cells, and 
the postsynaptic complex (anti-Shank1A) within the afferent dendrite. A double-blind strategy was employed for synapse quantification in confocal image stacks from 6 regions $\left(900 \mu \mathrm{m}^{2}\right.$ each) of medial extrastriola in flight and control utricles ( $n=4$ each). The central regions of flight and control horizontal cristae ( $n=4$ each) served as internal controls insensitive to static gravitational loading. Only complexes exhibiting colocalization of pre- and post- synaptic markers were quantified. We found that synapse densities in medial extrastriola hair cells decreased in flight utricles compared to controls. Flight and control cristae exhibited similar synapse densities, supporting the conclusion that utricular synapse density modifications resulted from unloading concomitant with microgravity exposure. These data further demonstrate the capability of utricular hair cells for synaptic plasticity resulting from alterations of the ambient sensory environment, though the specific modifications may be topology-dependent. 